\title{
Early postoperative MRI overestimates residual tumour after resection of gliomas with no or minimal enhancement
}

\author{
Sinan M. K. Belhawi • Friso W. A. Hoefnagels • Johannes C. Baaijen • \\ Esther Sanchez Aliaga • Jaap C. Reijneveld • Jan J. Heimans • Frederik Barkhof • \\ W. Peter Vandertop • Philip C. De Witt Hamer
}

Received: 27 September 2010 /Revised: 16 December 2010/Accepted: 10 January 2011 / Published online: 18 February 2011

(C) The Author(s) 2011. This article is published with open access at Springerlink.com

\begin{abstract}
Background Standards for residual tumour measurement after resection of gliomas with no or minimal enhancement have not yet been established. In this study residual volumes on early and late postoperative T2-/FLAIRweighted MRI are compared.

Methods A retrospective cohort included 58 consecutive glioma patients with no or minimal preoperative gadolinium enhancement. Inclusion criteria were first-time resection between 2007 and 2009 with a T2-/FLAIR-based target volume and availability of preoperative, early $(<48 \mathrm{~h})$ and late (1-7 months) postoperative MRI. The volumes of nonenhancing T2/FLAIR tissue and diffusion restriction areas were measured.

Results Residual tumour volumes were $22 \%$ smaller on late postoperative compared with early postoperative T2weighted MRI and 49\% smaller for FLAIR-weighted imaging. Postoperative restricted diffusion volume correlated with the difference between early and late postoperative
\end{abstract}

S. M. K. Belhawi • F. W. A. Hoefnagels · J. C. Baaijen •

W. P. Vandertop - P. C. De Witt Hamer

Neurosurgical Center Amsterdam, VU University Medical Center,

Amsterdam, The Netherlands

E. Sanchez Aliaga $\cdot$ F. Barkhof

Department of Radiology, VU University Medical Center,

Amsterdam, The Netherlands

J. C. Reijneveld · J. J. Heimans

Department of Neurology, VU University Medical Center,

Amsterdam, The Netherlands

P. C. De Witt Hamer $(\varangle)$

Department of Neurosurgery, Room 2F010, VU Medical Center, PO Box 7057, 1007 MB Amsterdam, The Netherlands

e-mail: p.dewitthamer@vumc.nl
FLAIR volumes and with the difference between $\mathrm{T} 2$ and FLAIR volumes on early postoperative MRI.

Conclusion We observed a systematic and substantial overestimation of residual non-enhancing volume on MRI within $48 \mathrm{~h}$ of resection compared with months postoperatively, in particular for FLAIR imaging. Resection-induced ischaemia contributes to this overestimation, as may other operative effects. This indicates that early postoperative MRI is less reliable to determine the extent of non-enhancing residual glioma and restricted diffusion volumes are imperative.

Keywords Glioma · Brain · Neurosurgical procedures · Magnetic resonance imaging . Diagnostic techniques and procedures $\cdot$ Diffusion magnetic resonance imaging

\section{Introduction}

Initial treatment for gliomas usually consists of resective surgery in order to obtain a histopathological diagnosis and to accomplish as radical a glioma removal as possible with preservation of brain functions. Important determinants of survival in gliomas are histopathological grading, patient age and clinical condition before treatment [1,2]. Residual tumour after resection is an independent prognostic factor for survival of low-grade and high-grade gliomas [3-6]. Therefore, a reliable quantitative measure of residual tumour after resection is important to accurately evaluate the impact of surgery on the course of the disease and to compare the extent of resection between patient series [6, 7].

For high-grade gliomas residual tumour is usually determined by tumour volume calculation on MRI of the elements that enhance after administration of gadolinium on T1-weighted imaging. To avoid artefacts from enhancing gliotic tissue in the resection margins and from adjuvant 
radiotherapy that can complicate delineation of residual tumour, the postoperative MRI is performed within $48 \mathrm{~h}$ of surgery [8]. Gliomas may also present with no or minimal gadolinium enhancement on MRI. A lower grade glioma is then suspected and the target volume for resection is concordantly based on the non-enhancing elements that are hyperintense on T2-weighted sequences. FLAIRweighted sequences have been particularly useful for this purpose, because the contrast between tumour and normal brain is superior to $\mathrm{T} 2$-weighted sequences as the signal from cerebrospinal fluid is suppressed [9]. Standards for the timing of postoperative MRI of gliomas with no or minimal enhancement have however not been established. The effects of the timing of postoperative MRI and of the pulse-sequence on residual tumour measurements have not been determined. We hypothesised that resection-induced ischaemia might contribute to differences in residual tumour volumes.

This study aimed to compare residual tumour volumes measured on early versus late postoperative T2- and FLAIRweighted MRI after resection of gliomas that show no or minimal gadolinium enhancement on preoperative imaging.

\section{Materials and methods}

\section{Inclusion criteria}

All adult patients with a histopathological diagnosis of a glioma grade 1 to 4 according to the WHO 2007 criteria [10], who had surgery between January 2007 and December 2009 were retrieved from a consecutive patient series in an electronic database of our institution, which is a tertiary referral centre for brain tumour treatment. Then, patients with first-time resective surgery were selected for analysis, excluding stereotactic or open biopsies. In order to ensure that the target volume for resection was based on T2-1 FLAIR-weighted imaging, only gliomas with no or minimal gadolinium enhancement on preoperative T1-weighted imaging were selected. However, focal or faint enhancement arbitrarily up to $15 \%$ of the T2/FLAIR volume, as determined volumetrically, was allowed. Furthermore, MR imaging was required to be available (a) preoperatively, (b) within $48 \mathrm{~h}$ of surgery, i.e. early postoperatively, and (c) between 2 and 6 months after surgery, i.e. late postoperatively.

The local ethical committee approved the research protocol and waived informed consent of participants.

\section{Resective surgery}

The patients in this study were preoperatively considered to have either a low-grade glioma or an anaplastic focus in a previously low-grade glioma. Therefore, the target volume for resection consisted of the T2/FLAIR volume. To minimise the risk of neurological morbidity this target volume was restricted by eloquent brain areas as determined by intraoperative cortical and subcortical electrostimulation mapping. The relation between the tumour and eloquent areas was determined according to the definitions used in the prognostic classification system of Chang et al [2]. Preoperative functional MRI, magnetic source imaging and diffusion tensor imaging tractography were obtained, as required by the location of the tumour. In general, the resection proceeded until eloquent brain areas were reached or volumetric tumour resection was complete. The tumour was subpially resected along sulci and fissures to minimise ischaemia around the resection cavity.

\section{MRI data acquisition}

MR imaging was performed on a $1.5 \mathrm{~T}$ system (Siemens Sonata or Avanto; Siemens Medical Systems, Erlangen, Germany). The imaging protocol included non-enhanced axial T1-weighted spin echo images [repetition time/echo time (TR/TE) 520-600/8-12 ms] with 5-mm section thickness and axial T2-weighted turbo spin echo images (TR/TE 5190-8670/93-101 ms) with 5-mm section thickness. Sagittal 3D turbo fluid-attenuated inversion-recovery (tFLAIR) images [repetition time/echo time/inversion time (TR/TE/TI) 6500/355/2200 ms] with $1.3-\mathrm{mm}$ section thickness plus axial multiplanar reconstructions (MPR) and axial single shot spin echo echo-planar diffusionweighted (DWI) images (TR/TE 3400/122 ms) with 5-mm section thickness were added to the imaging protocol in 2009. Diffusion gradients were applied along 3 orthogonal directions using b-values of 0,500 and $1000 \mathrm{~s} / \mathrm{mm}^{2}$. Apparent diffusion coefficient (ADC) maps were calculated from the DWI images. Post-contrast $(0.2 \mathrm{mmol} / \mathrm{Kg})$ sagittal 3D T1-weighted gradient-echo (MPRAGE) images (TR/ TE/TI 2300-2700/5-4.5/950 ms) with 1- to $1.5-\mathrm{mm}$ section thickness and axial T1-weighted spin echo images with 5-mm section thickness were obtained. In all patients preoperative imaging was repeated at our institution for navigation protocol purposes, even if preoperative imaging was available from a referring hospital.

\section{MRI volumetry}

Tumour volumes were measured using image fusion and volumetric software (BrainLab iPlan Cranial 2.6; BrainLab AG, Feldkirchen, Germany). MRI volumetry was based on the Cavalieri principle which provides unbiased volume estimates [11]. For this purpose tumour contours were manually segmented on sequential axial images and verified in the coronal and sagittal reconstruction planes. 
The sum of tumour contour surfaces of an MRI study was multiplied by slice thickness to obtain the estimated volume in $\mathrm{cm}^{3}$. This method has been demonstrated to be reproducible and accurate $[12,13]$. In this way preoperative tumour volumes were determined for T2-, FLAIRand gadolinium-enhanced T1-weighted images. The residual tumour after resection was also determined on T2- and FLAIR-weighted sequences for early and late postoperative imaging. The postoperative ischaemic volume was measured on an early postoperative diffusion-weighted sequence. Tumour and ischaemia were manually segmented independently by two observers (SB, PW) and disagreement was resolved by consensus with a third observer (ES).

\section{Comparison of volume measurements}

Several volume measurements were compared. To determine the effect of MRI timing on tumour volume measurements, early and late postoperative volumes were compared for T2- and FLAIR-weighted MRI. To determine the effect of pulse-sequence on tumour volume assessment, T2 and FLAIR volumes were compared on preoperative, early and late postoperative MRI. To determine the effect of resectioninduced ischaemia on differences in residual tumour volumes, tissue volumes with restricted diffusion on early postoperative MRI were compared with the difference between early and late postoperative volumes for T2- and FLAIR-weighted imaging.

\section{Statistical analysis}

Tumour volumes for individual patients were plotted for visual inspection. In the case of perfect agreement between two volumes, a linear regression coefficient of 1.0 with an intercept of 0 was expected (plotted as a dashed line) with a correlation coefficient of 1.0. The actual regression coefficients with $95 \%$ confidence intervals were calculated by linear regression analysis with a fixed intercept of 0 . As the volumes were considered not to be normally distributed, Spearman's correlation coefficient was calculated. Bland-Altman plots were created to visualise agreement between volume estimates. Absolute volume differences were compared using the Wilcoxon signed rank test for paired samples.

\section{Results}

Patient and MRI characteristics and histopathology

The study population was a subset of 58 patients out of 223 with a glioma diagnosis in the inclusion time interval.
Table 1 lists the details of the study population, consisting of 25 women and 33 men with a mean age of 44.7 years (range 20 to 70 ).

Preoperative MRI was performed at a median of 23 days (range: 1 to 282) before resection which showed no T1 gadolinium enhancement in 32 and minimal enhancement in 26 patients. The median preoperative $\mathrm{T} 2$ and FLAIR tumour volumes were $80.7 \mathrm{~cm}^{3}$ (range: 0.8 to 288.2 ) and $77.9 \mathrm{~cm}^{3}$ (range: 1.3 to 344.5 ), respectively. Histopathological diagnosis established WHO grade 1 in 2 patients $(3 \%)$, grade 2 in $35(60 \%)$, grade 3 in $14(24 \%)$ and grade 4 in $7(12 \%)$. Histopathological subtypes included 2 pilocytic astrocytomas (WHO grade 1); 14 astrocytomas, 18 oligodendrogliomas and 3 oligoastrocytomas (WHO grade 2); 6 anaplastic astrocytomas, 5 anaplastic oligodendrogliomas, 3 anaplastic oligoastrocytomas (WHO grade 3) and 7 glioblastomas (WHO grade 4).

Early and late postoperative T2 images were available for 52 patients; early and late postoperative FLAIR images for 14 patients. Early postoperative T2 and FLAIR images were available for 23 patients; late postoperative T2 and FLAIR images for 24 patients. Early postoperative diffusion-weighted images were available for 33 patients. Early postoperative MRI was obtained within 48 hours of resection in all patients, 44 on the first postoperative day, 14 on the second day. Late postoperative MRI was performed at a median of 100 days (range: 34 to 192) after resection; 18 within 3 months postoperatively, 31 between four and 6 months and 7 between seven and 9 months.

Residual tumour volumes on early compared with late postoperative MRI

The median early and late T2 residual volumes were $28.7 \mathrm{~cm}^{3}$ and $22.4 \mathrm{~cm}^{3}$, respectively, resulting in a $22 \%$ smaller residual volume on late T2 images. Residual T2 tumour volumes on early postoperative MRI demonstrated a systematically larger residual tumour volume (on average $4.3 \mathrm{~cm}^{3}$ ) compared with late postoperative MRI (Fig. 1a, c) with a regression coefficient of $0.767(95 \% \mathrm{CI}$ : $0.665-0.870)$, a correlation coefficient of $0.81(p<0.0001)$ and a paired Wilcoxon rank sum of $V=746.5(p=0.295)$. Similarly, the median early and late FLAIR residual volumes were $27.3 \mathrm{~cm}^{3}$ and $13.9 \mathrm{~cm}^{3}$, respectively, resulting in a $49 \%$ smaller residual volume on late FLAIR images. Systematically larger residual FLAIR tumour volumes (on average $5.7 \mathrm{~cm}^{3}$ ) were observed based on early postoperative MRI compared with late postoperative MRI (Fig. 1b, d) with a regression coefficient of 0.833 (95\%CI: 0.693-0.973), a correlation coefficient of 0.95 $(p<0.0001)$ and a paired Wilcoxon rank sum of $V=78(p=$ $0.119)$. Data plots confirmed that the differential residual 
Table 1 Patient demographics and MRI characteristics

\begin{tabular}{|c|c|c|c|c|c|}
\hline & WHO1 & WHO2 & WHO3 & WHO4 & total \\
\hline n $(\%)$ & $2(3 \%)$ & $35(60 \%)$ & $14(24 \%)$ & $7(12 \%)$ & 58 \\
\hline \multicolumn{6}{|l|}{ patient demographics } \\
\hline age, mean (SE) & $36.0(0.4)$ & $45.4(1.7)$ & $45.2(3.7)$ & $42.8(7.0)$ & $44.7(1.6)$ \\
\hline female/male & $0 / 2$ & $18 / 17$ & $5 / 9$ & $1 / 6$ & $25 / 33$ \\
\hline No. pts with radiotherapy & 0 & 2 & 6 & 2 & 10 \\
\hline No, pts with chemo-irradiation & 0 & 0 & 2 & 5 & 7 \\
\hline \multicolumn{6}{|l|}{ tumour lateralisation and location } \\
\hline $\mathrm{L} / \mathrm{R}$ & $0 / 2$ & $15 / 20$ & $5 / 9$ & $3 / 4$ & $23 / 35$ \\
\hline $\begin{array}{l}\text { nr pts with eloquent } \\
\text { tumour location }\end{array}$ & 2 & 26 & 7 & 6 & 41 \\
\hline frontal & 0 & 8 & 7 & 1 & 16 \\
\hline SMA & 0 & 7 & 2 & 2 & 11 \\
\hline parietal & 0 & 6 & 3 & 2 & 11 \\
\hline temporal & 0 & 2 & 1 & 1 & 4 \\
\hline insula & 2 & 12 & 1 & 1 & 16 \\
\hline \multicolumn{6}{|l|}{ MRI characteristics } \\
\hline $\begin{array}{l}\text { preoperative } \mathrm{T} 2 \text { hyperintense } \\
\text { tumour volume in } \mathrm{cm}^{3}, \\
\text { mean }(\mathrm{SE})\end{array}$ & $135.0(58.2)$ & $71.6(8.7)$ & $129.1(19.8)$ & $76.6(12.2)$ & $88.3(8.0)$ \\
\hline $\begin{array}{l}\text { preoperative FLAIR } \\
\text { hyperintense tumour volume } \\
\text { in } \mathrm{cm}^{3} \text {, mean (SE) }\end{array}$ & $129.9(52.0)$ & $72.3(9.9)$ & 138.7 (29.9) & $89.8(16.2)$ & $90.3(9.7)$ \\
\hline $\begin{array}{l}\text { days preoperative MRI, } \\
\text { mean (SE) }\end{array}$ & $162(121)$ & $56(15)$ & $27(9)$ & $38(14)$ & $50(10)$ \\
\hline $\begin{array}{l}\text { days early postoperative MRI, } \\
\text { mean (SE) }\end{array}$ & $1(0)$ & $1.4(0.1)$ & $1.8(0.3)$ & $1.6(0.4)$ & $1.5(0.1)$ \\
\hline $\begin{array}{l}\text { days late postoperative MRI, } \\
\text { mean (SE) }\end{array}$ & $189(10)$ & $104(7)$ & $117(21)$ & $96(15)$ & $109(7)$ \\
\hline
\end{tabular}

tumour volumes were independent of glioma grading, subtyping and timing of late postoperative MRI.

Tumour volumes on FLAIR- compared with T2-weighted imaging

FLAIR tumour volumes were marginally larger (on average $4.7 \mathrm{~cm}^{3}$ ) compared with $\mathrm{T} 2$ volumes on preoperative MRI (Fig. 2a, d) with a regression coefficient of $1.068(95 \%$ CI: $1.033-1.102)$, a correlation coefficient of $0.98(p<0.0001)$ and a paired Wilcoxon rank sum of $V=419.5(p=0.056)$. FLAIR residual tumour volumes were substantially larger (on average $7.2 \mathrm{~cm}^{3}$ ) than $\mathrm{T} 2$ tumour volumes on early postoperative MRI (Fig. 2b, e) with a regression coefficient of 1.156 (95\%CI: 1.049-1.262), a correlation coefficient of $0.96(p<0.0001)$ and a paired Wilcoxon rank sum of $V=40$ $(p=0.009)$. On the late postoperative MRI, FLAIR and T2 residual tumour volumes were comparable (Fig. 2c, f): T2 tumour volumes on average $1.5 \mathrm{~cm}^{3}$ larger with a regression coefficient of $0.948(95 \% \mathrm{CI}: 0.880-1.015)$, a correlation coefficient of $0.94(p<0.0001)$ and a paired Wilcoxon rank sum of $V=194(p=0.218)$. Again, data plots confirmed that the differential residual tumour volumes were independent of glioma grading and timing of late postoperative MRI.
Postoperative ischaemia

The median restricted diffusion volume was $6.1 \mathrm{~cm}^{3}$. The restricted diffusion volume only weakly correlated with the difference between early and late postoperative volumes on T2-weighted imaging (Fig. 3a) with a correlation coefficient of $0.32(p=0.089)$. The restricted diffusion volume however did correlate more strongly with the difference between early and late postoperative volumes on FLAIRweighted imaging (Fig. 3b) with a regression coefficient of 0.877 (95\%CI: 0.486-1.267) and a correlation coefficient of $0.76(p=0.004)$. In addition, the restricted diffusion volume also correlated with the difference between FLAIR and $\mathrm{T} 2$ volumes on early postoperative MRI (Fig. 3c) with a regression coefficient of 0.655 (95\%CI: $0.440-0.871)$ and a correlation coefficient of $0.77(p=0.0001)$.

\section{Discussion}

This study population consisted of a consecutive series of patients in whom resective surgery targeted a FLAIR-based tumour volume. Owing to the absence of overt T1 gadolinium enhancement these lesions were deemed low- 
Fig. 1 Residual tumour volumes in $\mathrm{mL}$ comparing early and late postoperative MRI based on T2weighted imaging and FLAIRweighted imaging shown as data plots (a and b) and BlandAltman plots (c and d) respectively depicting systematic overestimation of residual tumour volume by early postoperative MRI. Each data point represents measurements obtained from one patient, tagged by glioma grade according to the legend. The straight diagonal line in $\mathbf{a}$ and $\mathbf{b}$ represents hypothetical perfect agreement and the dotted lines the actual linear regression fit and corresponding $95 \%$ confidence interval. The three dotted horizontal lines in $\mathbf{c}$ and $\mathbf{d}$ represent the average differential volume and corresponding 95\% confidence interval
A

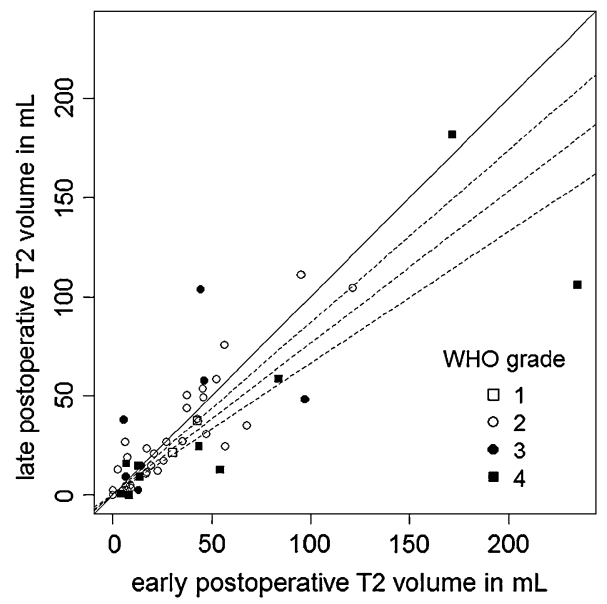

C

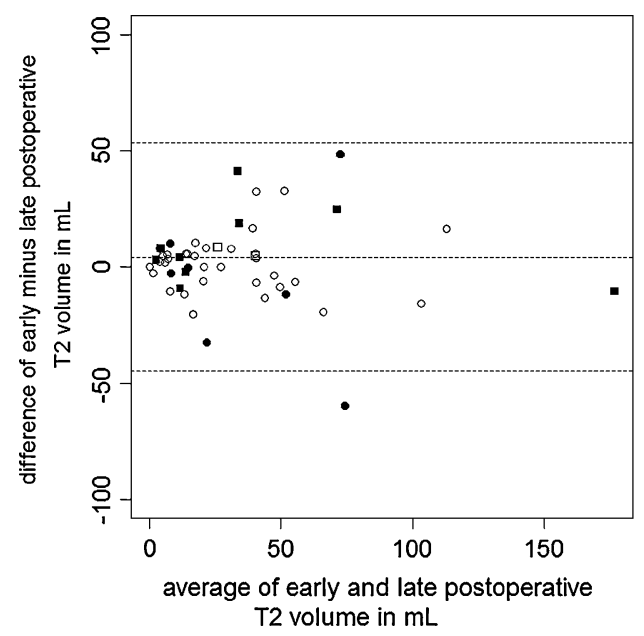

B

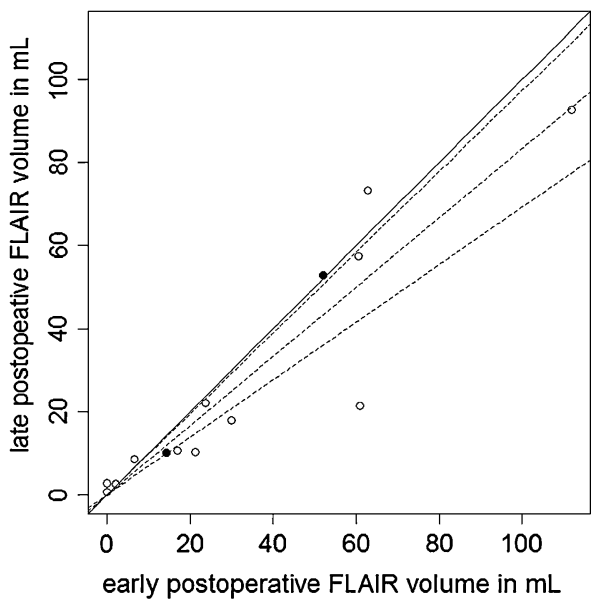

D

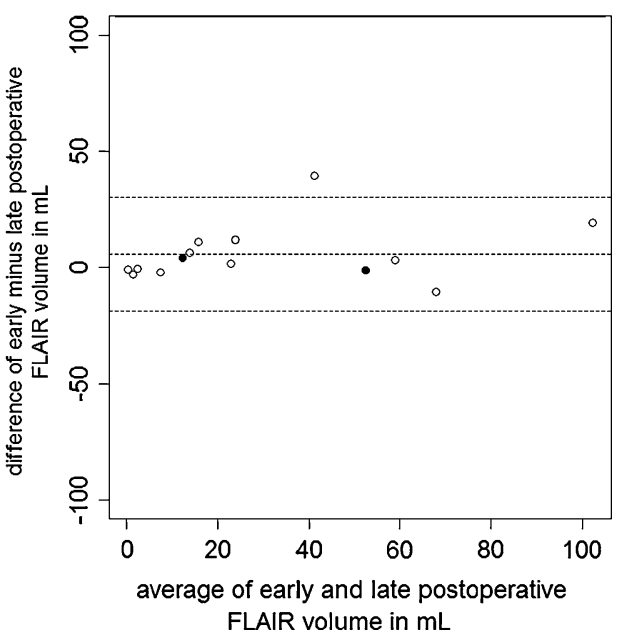

grade based on preoperative diagnostic neuroimaging. The histopathological diagnosis however established that 21 $(36 \%)$ of the gliomas were in fact of high grade (WHO grade 3 or 4), reflecting the well-known discordance between MRI and histopathological grading of glioma [14-16]. Three main observations were made in this study. First, a systematic and substantial overestimation of residual tumour on MRI within $48 \mathrm{~h}$ of resection, indicating that late MRI months after surgery is more suitable to determine the extent of resection than an early MRI days after resection. Second, our data do not support a preference for either FLAIR- or T2-weighted imaging for either pre- or late postoperative imaging, although FLAIR-weighted imaging is more sensitive for early postoperative overestimation than T2-weighted imaging. Third, resection-induced diffusion restriction contributes to the residual volume overestimation of early compared with late postoperative MRI. These main findings are illustrated with a patient example in Fig. 4.

Several factors likely contribute to the reduction of $\mathrm{T} 2 /$ FLAIR hyperintense regions between early and late postoperative MRI. It is unlikely that residual tumour actually regresses in the months following resection, although in theory involution of tumoural tissue induced by hypoperfusion due to surgical devascularisation can be postulated. The contribution of oedema and contusion of brain tissue surrounding the resection cavity, which subsides in weeks to months after resection, seems to be a more likely explanation. This reversible oedema can be either due to tumour compression that was present before resection, similar to peritumoral oedema as observed in some meningiomas for instance, or due to surgical manipulation of the tissue surrounding the resection cavity. Furthermore, the diffusion restricted areas may evolve in ischaemic (non-tumoral) tissue with volume involution over weeks to months similar to spontaneous infarction [17].

It is not surprising that $\mathrm{T} 2$ and FLAIR sequences provide similar tumour volumes on preoperative and late postoperative MRI because these sequences essentially measure the same physical properties of tissue except for the suppression of cerebrospinal fluid signal in the FLAIR sequence. This 

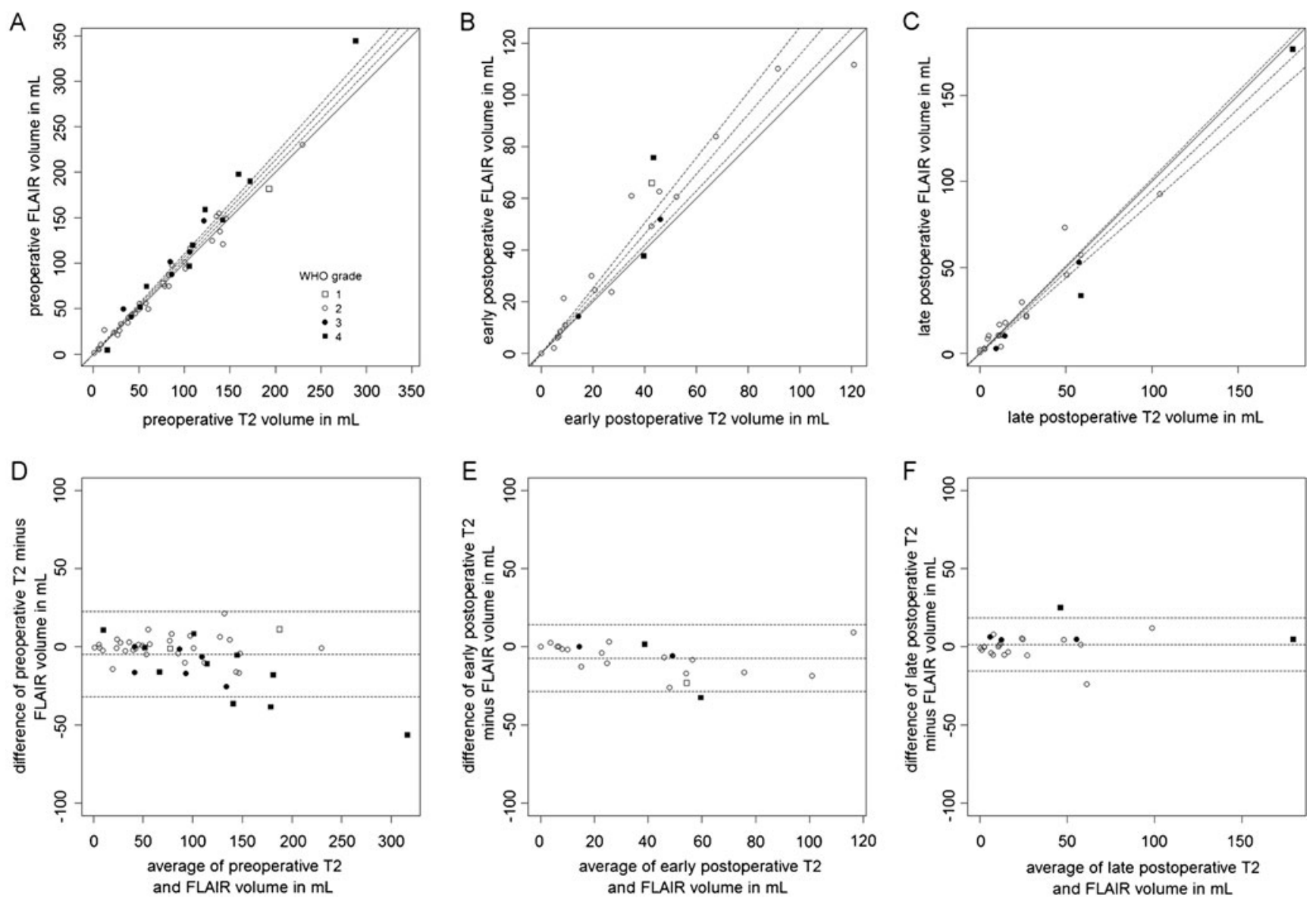

Fig. 2 Residual tumour volumes in $\mathrm{mL}$ comparing T2- and FLAIRweighted imaging respectively based on preoperative, early postoperative and late postoperative MRI shown as data plots $(\mathbf{a}, \mathbf{b}$ and $\mathbf{c})$ and Bland Altman plots (d, e and $\mathbf{f}$ ) depicting good agreement in residual tumour

suppression provides superior contrast among tumour, cerebrospinal fluid and brain, thereby facilitating for instance segmentation algorithms to delineate tumour volume in neuronavigation preplanning [18-20]. Nevertheless, FLAIR volume based on preoperative and late postoperative MRI and systematic overestimation of FLAIR-weighted imaging on early postoperative MRI. Data points, tagging and line styles as in Fig. 1

tumour volumes tend to be larger than T2 volumes on early postoperative MRI. This could be explained by differences in sensitivity in detecting ischaemia especially in grey matter [21-24] rather than by differences in the detection of residual
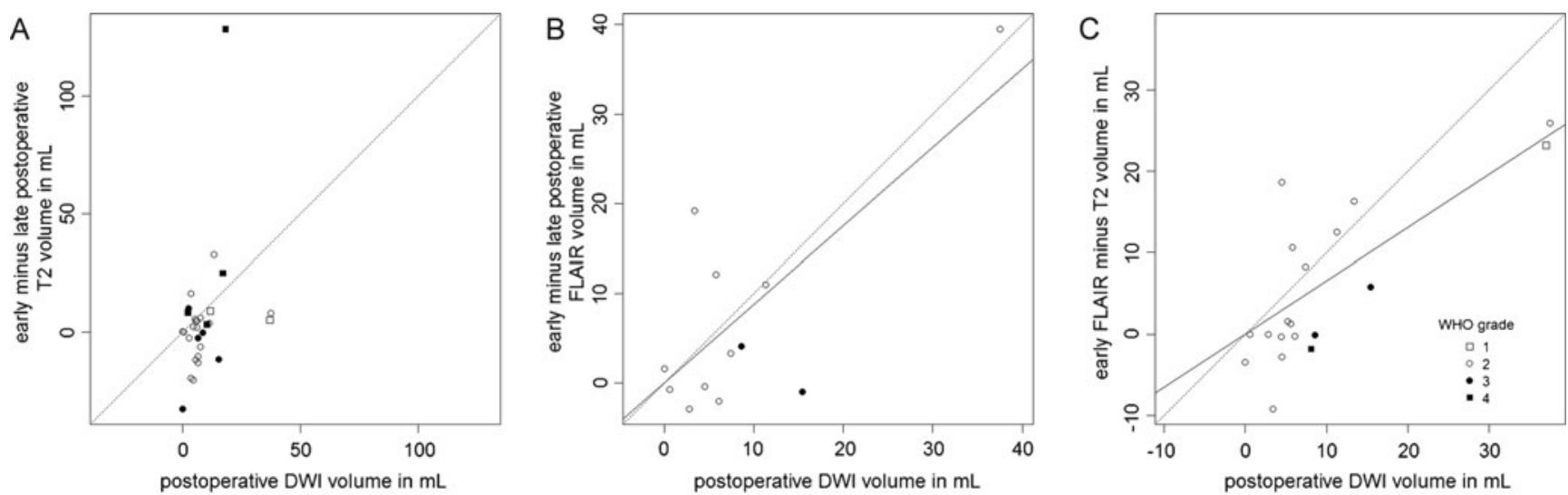

Fig. 3 Plots of diffusion restriction volume on early postoperative MRI and (a) the difference in T2 residual tumour volumes of early and late postoperative MRI, (b) the difference in FLAIR residual tumour volumes of early and late postoperative MRI and (c) the difference in early postoperative MRI residual tumour volumes of T2- and FLAIRweighted imaging. Data points, tagging and line styles as in Fig. 1 
preoperative
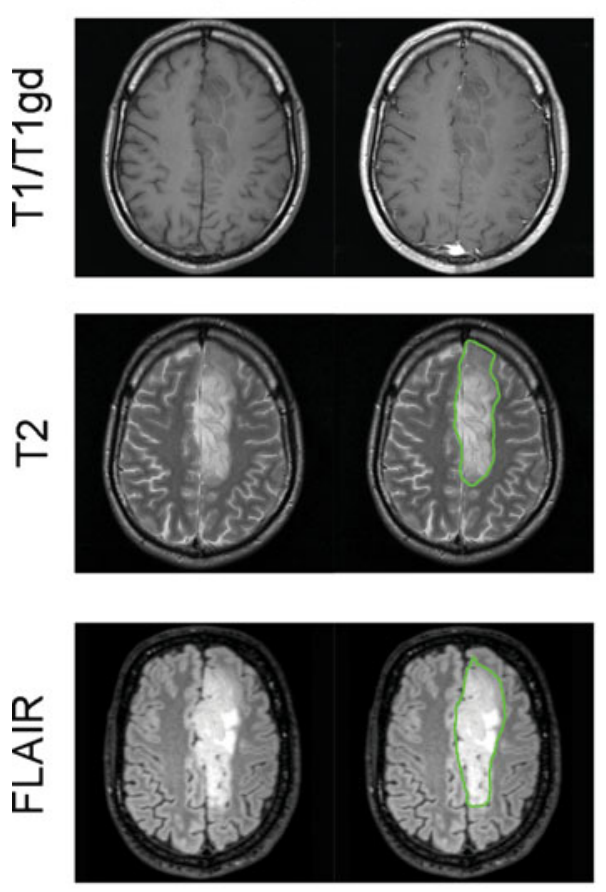

early postoperative

late postoperative
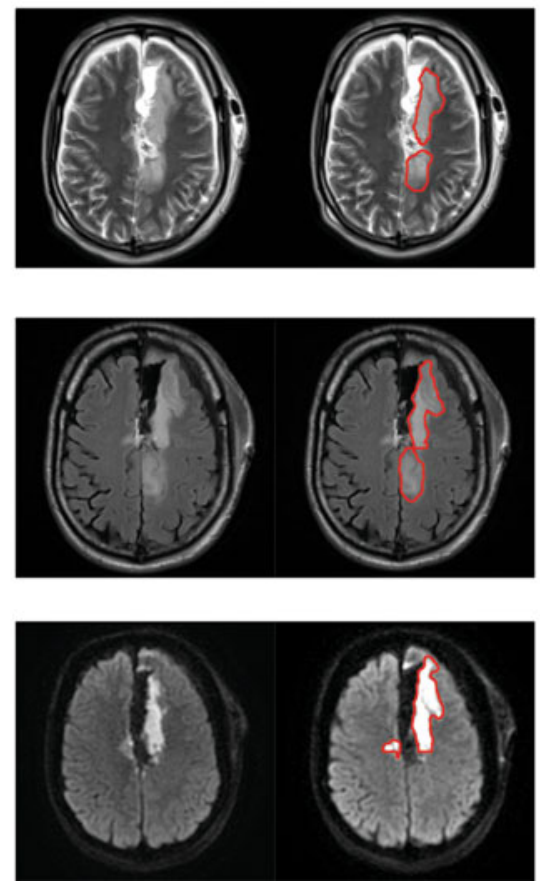
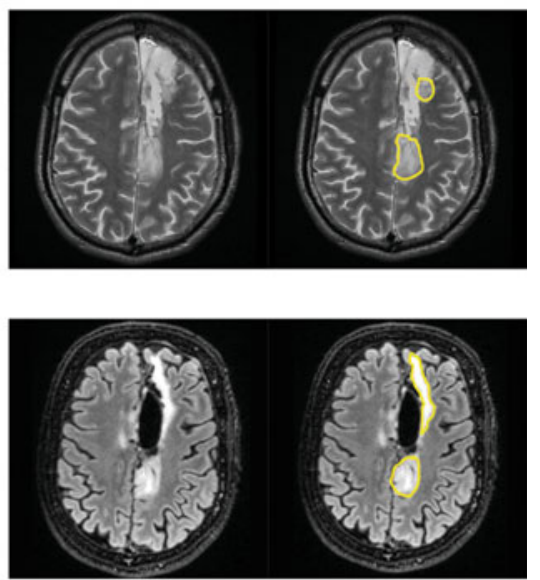

亏

volume of $37 \mathrm{~cm}^{3}$. The residual T2 and FLAIR volumes 97 days after resection measured 27 and $21 \mathrm{~cm}^{3}$, respectively, outlined in yellow. Note the involution of the hyperintensity at the lateral margin of the resection cavity with restricted diffusion, interpreted as resectioninduced ischaemia, and the stable hyperintensity at the posterior margin of the resection cavity, presumably genuine residual glioma within the corticospinal tract residual T2 and FLAIR volumes 2 days after surgery were 35 and $61 \mathrm{~cm}^{3}$, respectively, outlined in red, with a diffusion restriction

tumour or oedema originating from tumour compression or surgical manipulation.

Recent studies on the extent of resection of nonenhancing gliomas have used various postoperative MRI timings and sequences to determine residual tumour. Examples include using T2 or FLAIR MRI within $48 \mathrm{~h}$ postoperatively [25], MRI less than $48 \mathrm{~h}$ postoperatively and FLAIR-weighted imaging with subtraction of areas that were likely to contain oedema [3], unspecified postoperative timing of T2- or FLAIR-weighted MRI when available [26], unspecified timing of FLAIR-weighted MRI [4], T1weighted MRI at three to 6 months postoperatively [27], unspecified postoperative timing of T2- and FLAIRweighted MRI [28] and T2-weighted MRI at five or six weeks postoperatively [29]. In order to determine the impact of residual tumour on the course of disease and to be able to compare the extent of resection between patient series, it seems reasonable to use standardised objective criteria for measurements of residual tumour. Based on our observations, we recommend avoiding measuring the extent of resection on MRI immediately after resection. We also recommend incorporating diffusion-weighted imaging into the immediate postoperative MRI for distinction between residual tumour and ischaemia in the reporting of results on residual tumour after non-enhancing glioma resections.

Notwithstanding this limitation, early postoperative MRI is clearly useful for a number of other purposes. First, as several of these patients will be diagnosed with high-grade glioma, baseline imaging for response evaluation of adjuvant treatment is assured by early postoperative MRI. Second, incidental tumour progression between resection and late postoperative MRI can be detected only in 
comparison with an early postoperative MRI. Third, new gadolinium enhancement on late postoperative MRI can be recognised as ischaemia-related luxury perfusion rather than be misinterpreted as anaplastic transformation [30]. Fourth, immediate postoperative deficits can be placed in the context of proximity of the resection cavity to eloquent structures and resection-induced diffusion restriction of these structures. Fifth, it is likely that more educational benefit is obtained for the surgical team from early postoperative MRI with feedback information on the surgical decisions rather than from postoperative MRI months after resection.

One limitation of this study is that the timing of the late postoperative MRI was not standardised and serial late postoperative measurements are lacking. Therefore, the optimal timing for late postoperative MRI remains uncertain. Another limitation is that for only a subset of patients FLAIR-weighted imaging was available both early and late postoperatively. This is due to the fact that the FLAIR and DWI sequences were added to the routine imaging protocol in the last year of patient inclusion. Despite this potential lack of power, a systematic difference was detected between early and late postoperative residual volumes based on FLAIR-weighted imaging in 14 patients. A further limitation is that some patients were adjuvantly treated with radio- or chemotherapy protocolised by trial participation, which could potentially interfere with late residual tumour volumes. This would however result in an underestimation of the observed postoperative reduction of residual tumour volume.

As a consequence of the overestimation of residual tumour on early MRI, we propose that quantitation of extent of resection is preferably based on MRI months after resection. The optimal timing of postoperative MRI for standardized criteria is to be determined in future prospective studies. Furthermore, estimates of residual tumour based on early MRI are to be carefully distinguished from regions with resection-induced diffusion restriction to avoid undue underestimation of surgical treatment effects.

In conclusion, our observation of a systematic and substantial overestimation of residual non-enhancing volume on MRI within $48 \mathrm{~h}$ of resection compared with months after resection indicates that early postoperative MRI is less reliable for volumetry of non-enhancing residual glioma. Diffusionweighted imaging to detect resection-induced ischaemia is imperative in the interpretation of postresection tumour volumes.

Open Access This article is distributed under the terms of the Creative Commons Attribution Noncommercial License which permits any noncommercial use, distribution, and reproduction in any medium, provided the original author(s) and source are credited.

\section{References}

1. Gorlia T, van den Bent MJ, Hegi ME et al (2008) Nomograms for predicting survival of patients with newly diagnosed glioblastoma: prognostic factor analysis of EORTC and NCIC trial 2698122981/CE.3. Lancet Oncol 9:29-38

2. Chang EF, Smith JS, Chang SM et al (2008) Preoperative prognostic classification system for hemispheric low-grade gliomas in adults. J Neurosurg 109:817-824

3. McGirt MJ, Chaichana KL, Attenello FJ et al (2008) Extent of surgical resection is independently associated with survival in patients with hemispheric infiltrating low-grade gliomas. Neurosurgery 63:700-707, author reply 707-708

4. Smith JS, Chang EF, Lamborn KR et al (2008) Role of extent of resection in the long-term outcome of low-grade hemispheric gliomas. J Clin Oncol 26:1338-1345

5. Stummer W, Reulen HJ, Meinel T et al (2008) Extent of resection and survival in glioblastoma multiforme: identification of and adjustment for bias. Neurosurgery 62:564-576, discussion 564-576

6. Sanai N, Berger MS (2008) Glioma extent of resection and its impact on patient outcome. Neurosurgery 62:753-764, discussion 264-756

7. Berger MS, Deliganis AV, Dobbins J, Keles GE (1994) The effect of extent of resection on recurrence in patients with low grade cerebral hemisphere gliomas. Cancer 74:1784-1791

8. Albert FK, Forsting M, Sartor K, Adams HP, Kunze S (1994) Early postoperative magnetic resonance imaging after resection of malignant glioma: objective evaluation of residual tumor and its influence on regrowth and prognosis. Neurosurgery 34:45-60, discussion 60-41

9. Essig M, Metzner R, Bonsanto M et al (2001) Postoperative fluidattenuated inversion recovery MR imaging of cerebral gliomas: initial results. Eur Radiol 11:2004-2010

10. Louis DN, Ohgaki H, Wiestler OD, Cavenee WK (2007) WHO Classification of Tumours of the Central Nervous System(ed)^(eds), Fourth. World Health Organization

11. Mayhew TM, Olsen DR (1991) Magnetic resonance imaging (MRI) and model-free estimates of brain volume determined using the Cavalieri principle. J Anat 178:133-144

12. Sorensen AG, Patel S, Harmath C et al (2001) Comparison of diameter and perimeter methods for tumor volume calculation. $\mathrm{J}$ Clin Oncol 19:551-557

13. Shah GD, Kesari S, Xu R et al (2006) Comparison of linear and volumetric criteria in assessing tumor response in adult high-grade gliomas. Neuro Oncol 8:38-46

14. Barker FG 2nd, Chang SM, Huhn SL et al (1997) Age and the risk of anaplasia in magnetic resonance-nonenhancing supratentorial cerebral tumors. Cancer 80:936-941

15. Ginsberg LE, Fuller GN, Hashmi M, Leeds NE, Schomer DF (1998) The significance of lack of MR contrast enhancement of supratentorial brain tumors in adults: histopathological evaluation of a series. Surg Neurol 49:436-440

16. Scott JN, Brasher PM, Sevick RJ, Rewcastle NB, Forsyth PA (2002) How often are nonenhancing supratentorial gliomas malignant? A population study. Neurology 59:947-949

17. Ritzl A, Meisel S, Wittsack HJ et al (2004) Development of brain infarct volume as assessed by magnetic resonance imaging (MRI): follow-up of diffusion-weighted MRI lesions. J Magn Reson Imaging 20:201-207

18. Essig M, Knopp MV, Schoenberg SO et al (1999) Cerebral gliomas and metastases: assessment with contrast-enhanced fast fluid-attenuated inversion-recovery MR imaging. Radiology 210:551-557

19. Husstedt HW, Sickert M, Kostler H, Haubitz B, Becker H (2000) Diagnostic value of the fast-FLAIR sequence in MR imaging of intracranial tumors. Eur Radiol 10:745-752 
20. Bynevelt M, Britton J, Seymour H, MacSweeney E, Thomas N, Sandhu K (2001) FLAIR imaging in the follow-up of low-grade gliomas: time to dispense with the dual-echo? Neuroradiology 43:129-133

21. Siemonsen S, Mouridsen K, Holst B et al (2009) Quantitative t2 values predict time from symptom onset in acute stroke patients. Stroke 40:1612-1616

22. Ebinger M, Galinovic I, Rozanski M, Brunecker P, Endres M, Fiebach JB Fluid-attenuated inversion recovery evolution within 12 hours from stroke onset: a reliable tissue clock? Stroke 41:250-255

23. Thomalla G, Rossbach P, Rosenkranz M et al (2009) Negative fluid-attenuated inversion recovery imaging identifies acute ischemic stroke at 3 hours or less. Ann Neurol 65:724-732

24. Lansberg MG, Thijs VN, O'Brien MW et al (2001) Evolution of apparent diffusion coefficient, diffusion-weighted, and T2-weighted signal intensity of acute stroke. AJNR Am J Neuroradiol 22:637-644

25. Ahmadi R, Dictus C, Hartmann C et al (2009) Long-term outcome and survival of surgically treated supratentorial low-grade glioma in adult patients. Acta Neurochir (Wien) 151:1359-1365
26. Shaw EG, Berkey B, Coons SW et al (2008) Recurrence following neurosurgeon-determined gross-total resection of adult supratentorial low-grade glioma: results of a prospective clinical trial. J Neurosurg 109:835-841

27. Mandonnet E, Jbabdi S, Taillandier L et al (2007) Preoperative estimation of residual volume for WHO grade II glioma resected with intraoperative functional mapping. Neuro Oncol 9:63-69

28. Duffau H, Lopes M, Arthuis F et al (2005) Contribution of intraoperative electrical stimulations in surgery of low grade gliomas: a comparative study between two series without (198596) and with (1996-2003) functional mapping in the same institution. J Neurol Neurosurg Psychiatry 76:845-851

29. Scerrati M, Roselli R, Iacoangeli M, Pompucci A, Rossi GF (1996) Prognostic factors in low grade (WHO grade II) gliomas of the cerebral hemispheres: the role of surgery. J Neurol Neurosurg Psychiatry 61:291-296

30. Smith JS, Cha S, Mayo MC et al (2005) Serial diffusion-weighted magnetic resonance imaging in cases of glioma: distinguishing tumor recurrence from postresection injury. J Neurosurg 103:428-438 